\title{
The Faint-End of the Galaxy Luminosity Function in the Hydra I Cluster
}

\author{
H. Yamanoi ${ }^{1}$, M. Tanaka ${ }^{2}$, and Suprime-Cam Group \\ ${ }^{1}$ Department of Astronomical Science, School of Physical Sciences, The Graduate University \\ for Advanced Studies (Sokendai), National Astronomical Observatory of Japan \\ email: yamanoi.hitomi@nao.ac.jp \\ ${ }^{2}$ Department of Astronomy, School of Science, University of Tokyo
}

\begin{abstract}
We examine the galaxy luminosity functions (LFs) in the Hydra I cluster (Abell 1060) at $z=0.0126$, for which very faint galaxies (down to $M \sim-10$ ) have not been surveyed yet. We conclude that the total LF has a slightly steep slope $(\alpha \sim-1.6)$ at $-20<M<-10$ in the $B$ - and $R_{C}$-bands. The numbers of galaxies at the faint end $(M \gtrsim-14)$ differ in between the cluster center and the outskirts slightly. We divide the Hydra member galaxies into red/blue galaxies and find that the shape of LFs in the faint magnitude range is determined by red dwarf galaxies.
\end{abstract}

Keywords. galaxies, clusters, luminosity functions, dwarfs

\section{Introduction}

Dwarf galaxies account for a large share of cluster galaxies and should be closely related to the formation and evolution of clusters. Despite the obvious importance, however, the number of very faint (down to $M \sim-10$ ) cluster samples available to date is limited and properties of such faint galaxies remain unclear. In order to reveal properties of faint cluster dwarfs, we derive very faint-end slopes of LFs in the Hydra I cluster.

\section{Observations}

We base our analyses on the data taken with the Suprime-Cam on the Subaru telescope. A deep photometric survey was carried out in the $B$ - and $R_{C}$-bands. We observed the central region as well as a peripheral region of Hydra I to investigate the environmental dependence of colors of galaxies in the different regions of the cluster.

\section{Results and Discussion}

We find that the faint-end slope of total LFs in the Hydra is $\alpha \sim-1.6$ at $-20<M<$ -10 both in the $B$ - and $R_{C}$-bands. But, there is a small difference in the slopes of the Hydra LFs at the fainter range of $M \gtrsim-14$ in difference environments. The number of faint galaxies $(M>-14)$ increases in the cluster center. We then separate the Hydra member galaxies into red and blue galaxies. The shape of red LFs is similar to that of total LFs, while that of blue LFs is not. The Hydra I cluster is dominated by red dwarfs down to $M \sim-10$. We find the upturn of total and red LFs at $M \sim-14$. This upturn is also seen in several nearby clusters, but not in the field. We suggest that the upturn at the faint magnitudes is unique in cluster environments and it might reflect cluster formation histories. 